\title{
Refractory Plasmablastic Lymphoma
}

National Cancer Institute

\section{Source}

National Cancer Institute. Refractory Plasmablastic Lymphoma. NCI Thesaurus. Code C157684.

Plasmablastic lymphoma that is resistant to treatment. 